PROCEEDINGS OF THE

AMERICAN MATHEMATICAL SOCIETY

Volume 132, Number 9, Pages 2593-2601

S 0002-9939(04)07405-2

Article electronically published on March 25, 2004

\title{
A LOWER BOUND FOR THE BLOCH RADIUS OF $K$-QUASIREGULAR MAPPINGS
}

\author{
KAI RAJALA
}

(Communicated by Juha M. Heinonen)

\begin{abstract}
We give a quantitative proof to Eremenko's theorem (2000), which extends Bloch's classical theorem to the class of $n$-dimensional $K$-quasiregular mappings.
\end{abstract}

\section{INTRODUCTION}

Let $\Omega \subseteq \mathbb{R}^{n}$ be a domain. We call a mapping $f: \Omega \rightarrow \mathbb{R}^{n} K$-quasiregular, $1 \leq K<\infty$, if the coordinate functions of $f$ belong to $W_{l o c}^{1, n}(\Omega)$ and if

$$
\|D f(x)\|^{n} \leq K J_{f}(x)
$$

for almost every $x \in \Omega$. Here $\|\cdot\|$ is the operator norm and $J_{f}$ the Jacobian determinant of $f$. The definition of quasiregular mappings easily extends to mappings $f: \Omega \rightarrow \overline{\mathbb{R}}^{n}$, where $\overline{\mathbb{R}}^{n}$ is the one-point compactification of $\mathbb{R}^{n}$, equipped with the spherical metric, that is, the metric that makes the stereographic projection $\pi: \overline{\mathbb{R}}^{n} \rightarrow S^{n}\left(e_{n+1} / 2,1 / 2\right)$ an isometry. Here $S^{n}(p, r) \subseteq \mathbb{R}^{n+1}$ denotes the sphere with center $p$ and radius $r$. For calculations in $\overline{\mathbb{R}}^{n}$ it is more convenient to use the chordal metric $q$, defined by

$$
\begin{aligned}
q(x, y) & =\frac{|x-y|}{\sqrt{\left(1+|x|^{2}\right)\left(1+|y|^{2}\right)}}, \quad x, y \in \mathbb{R}^{n}, \\
q(x, \infty) & =\frac{1}{\sqrt{1+|x|^{2}}} .
\end{aligned}
$$

The chordal metric is $\pi / 2$-bi-Lipschitz equivalent to the spherical metric. Quasiregular mappings into $\overline{\mathbb{R}}^{n}$ are often called quasimeromorphic.

It has turned out that (non-constant) quasiregular mappings form a natural generalization of analytic functions to higher dimensions. In the late 1960s Reshetnyak showed that they are continuous, open and discrete, and that they preserve sets of measure zero. Reshetnyak's work initiated a systematic study of quasiregular mappings; see the monographs [15] and [16].

In 6] Eremenko generalized Bloch's classical theorem ([17, [2], [1] Chapter 1) for analytic functions, as well as a corresponding theorem for meromorphic functions by Minda [12] (see also [3]), to the class of $K$-quasiregular mappings.

Received by the editors April 15, 2003 and, in revised form, May 23, 2003.

2000 Mathematics Subject Classification. Primary 30C65.

(C)2004 American Mathematical Society 
Let $f: \Omega \rightarrow \mathbb{R}^{n}$ be a non-constant quasiregular mapping. For every $x \in \Omega$ define $d_{f}(x)$ as the radius of the maximal Euclidean open ball $B \subseteq \mathbb{R}^{n}$, centered at $f(x)$, such that a continuous right inverse $\phi$ with the property $\phi(f(x))=x$ exists in $B$. Note that by [4], the topological dimension of the branch set of $f$ is at most $n-2$, and thus such balls exist. The Bloch radius of $f$ is defined as

$$
\mathcal{B}_{e}(f)=\sup _{x \in \Omega} d_{f}(x)
$$

Similarly, we define the Bloch radius $\mathcal{B}_{s}(f)$ for quasimeromorphic mappings $f$ by looking at maximal open balls in the spherical metric. Notice that $\mathcal{B}_{s}(f) \leq \mathcal{B}_{e}(f)$ if $f$ is considered as the same mapping into $\mathbb{R}^{n}$ with either Euclidean or spherical metric. Recall that a family $\mathcal{F}$ of continuous mappings from an open and connected subset $\Omega$ of $\mathbb{R}^{n}$ into a metric space $Y$ is normal, if every sequence in $\mathcal{F}$ contains a subsequence converging uniformly on compact subsets of $\Omega$. If $Y$ is compact, $\mathcal{F}$ is normal if and only if its restriction to any compact subset of $\Omega$ is equicontinuous.

Eremenko's theorem now reads as follows:

Theorem 1.1 ([6], Theorem 1). (i) There exists a constant $b(n, K)>0$ such that the family of all $K$-quasimeromorphic mappings from the unit ball $B(0,1) \subseteq \mathbb{R}^{n}$ into $\overline{\mathbb{R}}^{n}$, whose spherical Bloch radii are at most $b(n, K)-\epsilon$, is normal for every $\epsilon \in(0, b(n, K))$.

(ii) For every non-constant $K$-quasimeromorphic mapping $f: \mathbb{R}^{n} \rightarrow \overline{\mathbb{R}}^{n}$ we have $\mathcal{B}_{s}(f) \geq b(n, K)$.

(iii) Every family of $K$-quasiregular mappings from the unit ball $B(0,1) \subseteq \mathbb{R}^{n}$ into $\mathbb{R}^{n}$ with bounded Bloch radius is equicontinuous with respect to the Euclidean metric.

(iv) Every non-constant $K$-quasiregular mapping $f: \mathbb{R}^{n} \rightarrow \mathbb{R}^{n}$ satisfies $\mathcal{B}_{e}(f)=$ $\infty$

Eremenko also gives an example showing that in any dimension $n \geq 3, b(n, K)$ depends on $K$ in Theorem 1.1. When $n=2, b(n, K)=b(2, K)$ does not depend on $K$, thanks to the Stoilow factorization theorem; see [9], page 241. It should also be noted that there exists a constant $K_{p}>1$ so that any non-constant $K$-quasiregular mapping $f: \Omega \rightarrow \mathbb{R}^{n}, \Omega \subseteq \mathbb{R}^{n}, n \geq 3$, with $K<K_{p}$ is a local homeomorphism; see [16, VI, Theorem 8.14. Moreover, every quasiregular local homeomorphism $f: \mathbb{R}^{n} \rightarrow \mathbb{R}^{n}, n \geq 3$, is actually a global homeomorphism by Zorich's theorem; see [16], III.3. Theorem 1.1 is related to the difficult question of describing the branching of quasiregular mappings. See [7] for a discussion on this topic.

Eremenko gives a normal family argument to prove Theorem 1.1] (see [10] for related results for normal families of quasiregular mappings). The proof is short and elegant, but it only gives the existence of the constant $b(n, K)$ without any estimates. The purpose of this paper is to give a quantitative proof for Theorem 1.1.

Theorem 1.2. One can choose $b(n, K)$ in Theorem [1.1] as $b(n, K)=\phi_{M}$, where $\phi_{M}$ is defined in the following way. Let $C_{1}$ and $C_{2}$ be the constants in Lemma 2.1] and inequality (2.5) below, depending only on $n$, and denote the $(n-1)$-dimensional 
Hausdorff measure of the unit sphere $S^{n-1}$ by $\omega_{n-1}$. Set

$$
\begin{gathered}
\alpha=K^{1 /(1-n)}, \quad \beta=\frac{\left(8 K^{n} \omega_{n-1} C_{1}^{-1}\right)^{1 /(n-1)}}{\log 2}-1, \\
\epsilon=2^{-6(1+1 / \alpha)-1} \exp \left(-\left(4 K^{n} \omega_{n-1} /\left(C_{1} \log \sqrt{3}\right)\right)^{1 /(n-1)}\right), \\
\eta=2^{-2 \beta-2} C_{2}^{\beta / \alpha} \epsilon^{\beta}, \quad m=\left(\frac{K \log \frac{4}{\eta}}{\log 2}\right)^{n-1},
\end{gathered}
$$

and let $M$ be the positive integer satisfying $\log _{2} m-1<M \leq \log _{2} m$. Define a function $\varphi:(0, \infty) \rightarrow(0, \infty)$ by

$$
\varphi(t)=\frac{t}{2} \exp \left(-\left(\frac{2 m K \omega_{n-1}}{C_{1} \log \left(1+t^{1 / \alpha} /\left(8^{1 / \alpha} \epsilon\right)\right)}\right)^{1 /(n-1)}\right) .
$$

Finally, set $\phi_{0}=\eta$, and for $i=0, \ldots, M-1$, define $\phi_{i+1}$ by $\phi_{i+1}=\varphi\left(\phi_{i}\right)$.

The estimate in Theorem 1.2 is far from being optimal. Still, the proof of Theorem 1.2 is constructive, at least after we have used a normal family method to restrict the consideration to mappings with desirable properties. Some of the ideas of the proof are from the proof of Rickman's theorem on omitted values of entire quasiregular mappings; see [16], Chapter IV.

\section{Preliminary Results}

We shall follow [16] as our basic reference for the theory of quasiregular mappings. We shall use the notation $M(\Gamma)$ for the usual $n$-modulus of a family of curves $\Gamma$. Chordal balls in $\overline{\mathbb{R}}^{n}$ are denoted by $Q(x, r)$, and Euclidean balls by $B(x, r)$. The corresponding notations for closed balls are $\bar{Q}(x, r)$ and $\bar{B}(x, r)$. The diameter of a set $E \subseteq \overline{\mathbb{R}}^{n}$ in the chordal metric is denoted by $q(E)$, while the corresponding notation for the Euclidean metric is $\operatorname{diam}(E)$. For the Euclidean distance between two sets $A_{1}$ and $A_{2}$ (or the distance between a point and a set), we use the notation $d\left(A_{1}, A_{2}\right)$.

Let us recall a useful modulus estimate.

Lemma 2.1 ([19], Theorem 10.12). Let $\Omega \subseteq \mathbb{R}^{n}$ be a domain, and $p \in \Omega$. Suppose that $0<a<b$ and that $E, F \subseteq \Omega$ are disjoint sets such that every sphere $S^{n-1}(p, t)$, $a<t<b$, meets both $E$ and $F$. If $\Omega$ contains the spherical ring $B(p, b) \backslash B(p, a)$ and if $\Gamma$ is the family of all curves joining $E$ and $F$ in $\Omega$, then

$$
M(\Gamma) \geq C_{1} \log \frac{b}{a},
$$

where $C_{1}$ only depends on $n$ (see [19], pages 28 and 31 for the precise value of $C_{1}$ ).

Recall that if $f: \Omega \rightarrow \overline{\mathbb{R}}^{n}$ is a continuous, open and discrete mapping and if $U \subset \subset \Omega$ is a domain, then $U$ is called a normal domain (for $f$ ) if $f \partial U=\partial f U$. Now we have the following:

Lemma 2.2 ([16], I, Lemma 4.7). If $V \subseteq \overline{\mathbb{R}}^{n}$ is a domain and if $U$ is a component of $f^{-1} V$ such that $U \subset \subset \Omega$, then $U$ is a normal domain and $f U=V \subset \subset f \Omega$.

As in [16], we will use the notation $U(x, f, s)$ for the $x$-component of the preimage $f^{-1}(Q(f(x), s))$. The notation $\mu(y, f, U)$ will be used for the topological degree of the mapping $f$ at $y \notin f(\partial U)$ with respect to the domain $U$. If $U$ is a normal 
domain, then $\mu(y, f, U)$ is constant for all $y \in f U$. We will denote this constant by $\mu(f, U)$.

For a quasiregular mapping $f: \Omega \rightarrow \mathbb{R}^{n}$ and a Borel set $E \subseteq \Omega$, define the counting function $n(E, y)$ by

$$
n(E, y)=\sum_{x \in f^{-1}(y) \cap E} i(x, f),
$$

where $i(x, f)$ is the local index. See [16], I.4 for information on the topological degree and the local index. The following modulus inequality will be useful for our purposes. Recall that a Borel function $\rho: \mathbb{R}^{n} \rightarrow[0, \infty]$ is said to be admissible for a curve family $\Gamma$ if it qualifies as a test function for the modulus $M(\Gamma)$, i.e., if

$$
\int_{\gamma} \rho d s \geq 1
$$

for all locally rectifiable non-constant curves $\gamma \in \Gamma$.

Lemma 2.3 (16], II, Theorem 2.4 and Remark 2.5). Let $f: \Omega \rightarrow \mathbb{R}^{n}$ be a $K$ quasiregular mapping, and suppose $E \subset \subset \Omega$ is a Borel set. If $\Gamma$ is a family of curves in $E$ and if $\rho$ is an admissible function for $f \Gamma$, then

$$
M(\Gamma) \leq K \int_{\mathbb{R}^{n}} \rho^{n}(y) n(E, y) d y .
$$

We will also use a comparison inequality for the counting function. For an $(n-1)$-dimensional sphere $Y=S^{n-1}(0, t) \subseteq \mathbb{R}^{n}$, define the average $\nu(E, Y)$ of the counting function $n(E, \cdot)$ over the sphere $Y$ by

$$
\nu(E, Y)=\frac{1}{\omega_{n-1}} \int_{S^{n-1}} n(E, t y) d y,
$$

where $S^{n-1}$ is the unit sphere. The definition extends to all spheres in $\mathbb{R}^{n}$ (as well as in $\overline{\mathbb{R}}^{n}$ ) by translation. We shall use the notation $\nu(r, t)$ if $E=B(x, r)$ and if the center points need not be emphasized. Now we have the following comparison result in terms of the Euclidean metric. Here $K_{I}(f)=K_{I} \leq K^{n-1}$ is the inner dilatation of $f$; see [16], page 11 .

Lemma 2.4 ([16], IV, Lemma 1.1). Let $f: \Omega \rightarrow \mathbb{R}^{n}$ be a $K$-quasiregular mapping. If $\theta>1$, if $r, s, t>0$, and if $\bar{B}(0, \theta r) \subseteq \Omega$, then

$$
\nu(\theta r, t) \geq \nu(r, s)-\frac{K_{I}|\log (t / s)|^{n-1}}{(\log \theta)^{n-1}},
$$

whenever the $t$ - and s-spheres have the same center point.

The normal family method, introduced by Zalcman [20, has turned out to be an important tool in complex function theory. This method was generalized to the class of quasimeromorphic mappings in higher dimensions by Miniowitz 13. We shall formulate Miniowitz's result as in [6]. Although the estimate (2.5) below is not explicitly stated in [13, Lemma 1, it can be isolated from the proof.

Lemma 2.5 (13], Lemma 1). Let $\mathcal{F}$ be a family of $K$-quasimeromorphic mappings from the unit ball $B(0,1) \subseteq \mathbb{R}^{n}$ into $\overline{\mathbb{R}}^{n}$ that is not normal. Then there exist $r \in(0,1)$ and sequences $\left(f_{m}\right)$ in $\mathcal{F},\left(x_{m}\right)$ in $B(0, r)$ and $\left(\rho_{m}\right), 0<\rho_{m} \rightarrow 0$, such that the following holds. If $\left(g_{m}\right)$ is a sequence of mappings defined by $g_{m}=f_{m} \circ h_{m}$, 
$h_{m}(x)=x_{m}+\rho_{m} x$, then $\left(g_{m}\right)$ converges locally uniformly to a non-constant $K$ quasimeromorphic mapping $f: \mathbb{R}^{n} \rightarrow \overline{\mathbb{R}}^{n}$. Moreover, we have that for every $R>0$ and every $x_{1}, x_{2} \in B(0, R)$,

$$
q\left(f\left(x_{1}\right), f\left(x_{2}\right)\right) \leq 2\left(1+R^{2}\right)^{\alpha}\left|x_{1}-x_{2}\right|^{\alpha},
$$

where $\alpha=K^{1 /(1-n)}$, and

$$
q(f(B(0,1))) \geq C_{2}^{1 / \alpha}>0, \quad \text { where }\left(32 \lambda_{n}\right)^{-1} \leq C_{2}<1,
$$

and $\lambda_{n} \leq 2 e^{n-1}$ is the Grötzsch ring constant (cf. [16], page 60).

What makes normal families useful here is the fact that the Bloch radius is lower semicontinuous with respect to locally uniform convergence.

Lemma 2.6 (6], page 559). Let $\left(g_{m}\right)$ be a family of $K$-quasimeromorphic mappings into $\overline{\mathbb{R}}^{n}$, and suppose that $g_{m} \rightarrow f$ uniformly on compacta. Then

$$
\mathcal{B}_{s}(f) \leq \liminf _{m} \mathcal{B}_{s}\left(g_{m}\right) .
$$

\section{Proof of Theorem 1.2}

We first show that mappings such as $f$ in Lemma2.5 behave well in some normal domains $U$.

Proposition 3.1. Let $f: \mathbb{R}^{n} \rightarrow \overline{\mathbb{R}}^{n}$ be a $K$-quasimeromorhic mapping for which the estimates (2.4) and (2.5) hold true. Then there exist $x \in B(0,1)$ and constants $\eta, \epsilon, m>0$, depending only on $n$ and $K$, so that

$$
U=U(x, f, \eta) \subseteq B(x, \epsilon) \subseteq B(0,1)
$$

is a normal domain and $\mu(f, U) \leq m$. The values of the constants $\eta, \epsilon$ and $m$ are given in the statement of Theorem 1.2.

Proof. Set $\theta=\exp \left(\left(4 K K_{I} \omega_{n-1} /\left(C_{1} \log \sqrt{3}\right)\right)^{1 /(n-1)}\right)$, where $C_{1}$ is as in Lemma 2.1 and $\epsilon=2^{-6(1+1 / \alpha)-1} / \theta$, where $\alpha$ is as in Lemma 2.5. Note that $\theta>1$ and $\epsilon<2^{-7}$. Let $x$ and $y$ be such that

$$
q(f(\bar{B}(0,1)))=q(f(x), f(y)) .
$$

Let $N$ be the smallest integer satisfying $N \geq \epsilon^{-1}$. Now the union of the two line segments $\{t x: t \in[0,1]\}$ and $\{t y: t \in[0,1]\}$ can be covered by closed $\epsilon$-balls $\bar{B}_{i}$, $i=1, \ldots, N$, so that $\bar{B}_{i} \subseteq \bar{B}(0,1)$ for every $i$. By the lower bound (2.5),

$$
C_{2}^{\frac{1}{\alpha}} \leq q(f(B(0,1))) \leq \sum_{i=1}^{N} q\left(f\left(B_{i}\right)\right) \leq N \max _{i} q\left(f\left(B_{i}\right)\right),
$$

and thus for some ball $B(x, \epsilon) \subseteq B(0,1)$ we have that

$$
q(f(B(x, \epsilon))) \geq \frac{C_{2}^{\frac{1}{\alpha}}}{N} \geq \frac{C_{2}^{\frac{1}{\alpha}} \epsilon}{2} .
$$

Denote

$$
r=\sup \{t: U(x, f, t) \subseteq B(x, \epsilon)\}
$$

and

$$
R=\inf \{t: f(B(x, \epsilon)) \subseteq Q(f(x), t)\} .
$$

By our choice of $\epsilon$ and the continuity estimate (2.4), we have

$$
R<q(f(B(x, \epsilon)))<q(f(B(x, 2 \epsilon)))<1 / 2 .
$$


Now the $x$-component $U_{r}$ of $f^{-1}(Q(f(x), r))$ has the properties $x \in U_{r}$ and $\bar{U}_{r} \cap$ $S^{n-1}(x, \epsilon) \neq \emptyset$. Also, we claim that $f^{-1}\left(\overline{\mathbb{R}}^{n} \backslash \bar{B}(f(x), R)\right)=: W$ has, for arbitrarily large $i$, components $U_{i}$ with

$$
S^{n-1}(x, 2 \epsilon) \cap U_{i} \neq \emptyset, \quad d\left(U_{i}, S^{n-1}(x, \epsilon)\right)<\frac{1}{i} .
$$

To see this we take a point $z \in W$ so that $d\left(z, S^{n-1}(x, \epsilon)\right)<1 / i$. This is possible by the definition of $R$ and by the fact that for all $x \in \mathbb{R}^{n}$ there exists a radius $r_{x}$ such that $U(x, f, s)$ is a normal domain for all $s \leq r_{x}$; see [16], I, Lemma 4.9. Then assume that the $z$-component $U_{i}$ of $W$ lies inside $B(x, 2 \epsilon)$. By Lemma 2.2 $f\left(U_{i}\right)=\overline{\mathbb{R}}^{n} \backslash Q(f(x), R)$, and in particular $q(f(B(x, 2 \epsilon)))=q\left(\overline{\mathbb{R}}^{n}\right)=1$. This is a contradiction. Thus the claim holds.

Consider the family $\Gamma$ of all curves joining $B(x, 2 \epsilon) \cap\left(\cup_{i} U_{i}\right)$ and $U_{r} \cap B(x, 2 \epsilon)$ in $B(x, 2 \epsilon)$. Then for all $i \in \mathbb{N}$ the inequality $M(\Gamma) \geq M\left(\Gamma_{i}\right)$ holds, where $\Gamma_{i}$ is the family of all curves joining $B(x, 2 \epsilon) \cap U_{i}$ and $U_{r} \cap B(x, 2 \epsilon)$ in $B(x, 2 \epsilon)$. Now a simple geometric argument (see 8], Lemma 3.1) shows that for all $i \in \mathbb{N}$ we can use Lemma 2.1 with $a=\epsilon / 2+1 / i, b=\sqrt{3} \epsilon / 2, \Omega=B(x, 2 \epsilon)$ and $p$ defined as follows: Pick points $v \in S^{n-1}(x, \epsilon) \cap \bar{U}_{r}$ and $z_{i} \in S^{n-1}(x, \epsilon)$ so that $d\left(z_{i}, U_{i}\right)<1 / i$. Now, if the angle $\angle\left(x,\left(z_{i}+x\right) / 2, v\right)<\pi / 2$, choose $p=\left(z_{i}+x\right) / 2$; otherwise choose $p=\left(z_{i}+v\right) / 2$. Thus

$$
M\left(\Gamma_{i}\right) \geq C_{1} \log \frac{\sqrt{3} \epsilon / 2}{\epsilon / 2+1 / i},
$$

for all $i \in \mathbb{N}$, and so

$$
M(\Gamma) \geq C_{1} \log \sqrt{3}
$$

By composing $f$ with a rotation, we may assume $f(x)=0$. Now, since by (2.4) $R \leq q(f(B(x, \epsilon))) \leq 2^{-5}$,

$$
\Lambda=\mathrm{Id}:(Q(0, R), q) \rightarrow(Q(0, R), d)
$$

is (at least) 2-bi-Lipschitz. Here the notation $d$ is used for the Euclidean metric. Thus using the Euclidean metric in calculating $M(f \Gamma)$ only changes the value by a multiplicative constant smaller than 2 . Let $\Lambda(Q(0, R))=B\left(0, R^{\prime}\right)$ and $\Lambda(Q(0, r))=$ $B\left(0, r^{\prime}\right)$.

Lemma 2.3 can now be used with the function

$$
\rho(y)= \begin{cases}\frac{1}{\left(\log \frac{R^{\prime}}{r^{\prime}}\right)|y|}, & r^{\prime}<|y|<R^{\prime}, \\ 0 & \text { elsewhere }\end{cases}
$$

(compare [16], page 82). Combining the estimates and using Lemma 2.4 with $r=2 \epsilon$, we have

$$
\begin{aligned}
C_{1} \log \sqrt{3} \leq & M(\Gamma) \leq 2 K\left(\log \frac{R^{\prime}}{r^{\prime}}\right)^{-n} \int_{B\left(0, R^{\prime}\right) \backslash \bar{B}\left(0, r^{\prime}\right)} n(2 \epsilon, y)|y|^{-n} d y \\
= & 2 \omega_{n-1} K\left(\log \frac{R^{\prime}}{r^{\prime}}\right)^{-n} \int_{r^{\prime}}^{R^{\prime}} \frac{\nu(2 \epsilon, s)}{s} d s \leq 2 \omega_{n-1} K\left(\log \frac{R^{\prime}}{r^{\prime}}\right)^{-n} \\
& \times \int_{r^{\prime}}^{R^{\prime}} s^{-1}\left(\nu\left(2^{-6(1+1 / \alpha)}, R^{\prime}\right)+K_{I}\left(\log \frac{R^{\prime}}{r^{\prime}}\right)^{n-1}(\log \theta)^{1-n}\right) d s \\
= & 2 \omega_{n-1} K\left(\log \frac{R^{\prime}}{r^{\prime}}\right)^{1-n} \nu\left(2^{-6(1+1 / \alpha)}, R^{\prime}\right)+2 K_{I} K \omega_{n-1}(\log \theta)^{1-n} .
\end{aligned}
$$


By our choice of $\theta$, the second term on the previous line is smaller than $\frac{1}{2} C_{1} \log \sqrt{3}$, and so we further have

$$
\left(\log \frac{R^{\prime}}{r^{\prime}}\right)^{n-1} \leq 8 K \omega_{n-1} C_{1}^{-1} \nu\left(2^{-6(1+1 / \alpha)}, R^{\prime}\right) .
$$

Since $B\left(x, 2^{-6(1+1 / \alpha)+1}\right) \subseteq B(0,2)$, (2.4) gives

$$
\operatorname{diam}\left((\Lambda \circ f)\left(B\left(x, 2^{-6(1+1 / \alpha)+1}\right)\right)\right)<<1 .
$$

Applying Lemma 2.4 again with $t=1$ and $s=R^{\prime}$ gives

$$
\begin{aligned}
\nu\left(2^{-6(1+1 / \alpha)}, R^{\prime}\right) & \leq \nu\left(2^{-6(1+1 / \alpha)+1}, 1\right)+K_{I}(\log 2)^{1-n}\left(\log \frac{1}{R^{\prime}}\right)^{n-1} \\
& =K_{I}(\log 2)^{1-n}\left(\log \frac{1}{R^{\prime}}\right)^{n-1} .
\end{aligned}
$$

Since $R^{\prime} \geq R \geq C_{2}^{\frac{1}{\alpha}} \epsilon / 4$, combining inequalities (3.2) and (3.4) gives

$$
\left(\log \frac{C_{2}^{1 / \alpha} \epsilon}{4 r^{\prime}}\right)^{n-1} \leq 8 K \omega_{n-1} C_{1}^{-1} K_{I}(\log 2)^{1-n}\left(\log \frac{4}{C_{2}^{1 / \alpha} \epsilon}\right)^{n-1}
$$

i.e.,

$$
r \geq \frac{1}{2} r^{\prime} \geq \frac{1}{2}\left(\frac{C_{2}^{1 / \alpha} \epsilon}{4}\right)^{\beta}=: 2 \eta, \text { where } \beta=\frac{\left(8 K \omega_{n-1} C_{1}^{-1} K_{I}\right)^{1 /(n-1)}}{\log 2}-1 .
$$

This tells us that whenever $t<2 \eta, \bar{U}(x, f, t)$ does not intersect $S^{n-1}(B(x, \epsilon))$, and thus by Lemma $2.2 U=U(x, f, t)$ is a normal domain in this case. This proves the first claim of the proposition.

For the second claim we need to show that the topological degree is bounded in $U$, with a bound depending only on $n$ and $K$. By (3.3), also

$$
\operatorname{diam}((\Lambda \circ f)(B(x, 2 \epsilon)))<<1,
$$

and using Lemma 2.4 as above gives

$$
\begin{aligned}
\nu(\epsilon, \eta / 4) & \leq \nu(2 \epsilon, 1)+\frac{K_{I}}{(\log 2)^{n-1}}\left(\log \frac{4}{\eta}\right)^{n-1} \\
& =\frac{K_{I}}{(\log 2)^{n-1}}\left(\log \frac{4}{\eta}\right)^{n-1}=: m .
\end{aligned}
$$

By the definition of the average $\nu$ and the correspondance between the topological degree and the local index (see [16, I.4), inequality (3.6) shows in particular that there exists a point $y \in Q(0, \eta)$ such that $\mu(y, f, U) \leq m$. On the other hand, since the topological degree is constant in a normal domain, we have $\mu(f, U) \leq m$. This proves the second claim of the proposition.

We need a topological lemma from [11. This lemma is closely related to Newman's theorem [14] on transformation groups. See 5], 44, [18, for related results. By a proper mapping we mean a mapping with the property that the preimage of an arbitrary compact set is compact.

Lemma 3.2 ([11, Theorem 2). Let $G$ be an open, connected, relatively compact subset of $\mathbb{R}^{n}$, and $Y$ an n-dimensional manifold (possibly with boundary). If $f: \bar{G} \rightarrow Y$ is a continuous, proper, finite-to-one open mapping that is not a homeomorphism, and if

$$
f^{-1}(\partial Y) \subseteq(\bar{G} \backslash G), \quad D=\max \{d(x, \bar{G} \backslash G): x \in G\},
$$


and

$$
C=\max \left\{\operatorname{diam}\left(f^{-1}(f(x))\right): x \in \bar{G} \backslash G\right\},
$$

then $D \leq C$.

Proof of Theorem 1.2. Since we are only after the constant $b(n, K)$, we do not consider (iii) and (iv) in Theorem 1.1. First, we argue as in [6] to show that for both $(i)$ and $(i i)$ in Theorem 1.1 it suffices to prove (ii) for mappings with the properties of $f$ in Lemma2.5. For $(i)$ this is clear by Lemmas 2.5 and 2.6. For $(i i)$ it suffices to notice that if $f: \mathbb{R}^{n} \rightarrow \overline{\mathbb{R}}^{n}$ is $K$-quasimeromorphic and non-constant, then the family $\left\{x \rightarrow f\left(2^{n} x\right): n \in \mathbb{N}\right\}$ cannot be a normal family.

Let $f, x, \eta, \epsilon, U$ and $m$ be as in Proposition 3.1. We shall use Lemma 3.2 for $f: U(x, f, \eta / 2) \rightarrow Q(f(x), \eta / 2)$ (here we denote the restriction also by $f$ ). Recall that we have a uniform continuity estimate (2.4) for $f$, and thus

$$
D \geq\left(\frac{\eta}{8}\right)^{1 / \alpha}=: T
$$

where $D$ is as in Lemma 3.2 Now by Lemma 3.2, we have a point $z_{1} \in \partial Q(f(x), \eta / 2)$ such that

$$
\operatorname{diam}\left(f^{-1}\left(z_{1}\right) \cap \bar{U}(x, f, \eta / 2)\right) \geq T
$$

(unless $f: U(x, f, \eta / 2) \rightarrow Q(f(x), \eta / 2)$ is a homeomorphism, which would complete the proof). We claim that there exists a constant $\phi_{1}=\phi_{1}(n, K)>0$ such that $f^{-1}\left(z_{1}\right) \cap \partial U(x, f, \eta / 2)$ cannot be contained in a single component of $f^{-1}\left(Q\left(z_{1}, r\right)\right)$ for $r \leq \phi_{1}$.

Suppose that the set $f^{-1}\left(z_{1}\right) \cap \partial U(x, f, \eta / 2)$ is contained in a single component $V$ of $f^{-1}\left(Q\left(z_{1}, r\right)\right)$ for $r<\eta / 4$. Then by (3.7), $\operatorname{diam}(V) \geq T$. Denote by $\Gamma$ the family of all curves joining $\partial V$ and $\partial U$ in $B(x, \epsilon)$. Since $U \subseteq B(x, \epsilon)$, it follows that $M(\Gamma) \geq M\left(\Gamma^{\prime}\right)$, where $\Gamma^{\prime}$ is the family of all curves joining $S^{n-1}(x, \epsilon)$ and $V$. For $M\left(\Gamma^{\prime}\right)$ we can apply Lemma 2.1 with $a=\epsilon, b=T+\epsilon$ and $p$ defined as follows: Let $y_{1}, y_{2} \in \partial V$ be points such that $\operatorname{diam}(V)=d\left(y_{1}, y_{2}\right)$. Now one can define $p$ to be either of the two points on the line passing through $y_{1}$ and $y_{2}$, with distance $\epsilon$ from the line segment $\left\{t y_{1}+(1-t) y_{2}: t \in[0,1]\right\}$.

On the other hand, $U$ and $V$ are normal domains, and so $M(f \Gamma) \leq M\left(\Gamma^{*}\right)$, where $\Gamma^{*}$ is the family of all curves joining $Q(z, r)$ and $Q(z, \eta / 2)$. As in the proof of Proposition 3.1, we can use the 2-bi-Lipschitz property of $\Lambda$ in order to estimate the modulus by calculating in the Euclidean metric. Combining with Lemma 2.3 (notice again the correspondance between the topological degree and the local index), we have

$$
\begin{aligned}
C_{1} \log \left(1+\frac{T}{\epsilon}\right) & \leq M\left(\Gamma^{\prime}\right) \leq M(\Gamma) \leq m K M(f \Gamma) \leq m K M\left(\Gamma^{*}\right) \\
& \leq 2 \omega_{n-1} m K\left(\log \frac{\eta}{2 r}\right)^{1-n} .
\end{aligned}
$$

This is a contradiction for

$$
r \leq \frac{\eta}{2} \exp \left(-\left(\frac{2 m K \omega_{n-1}}{C_{1} \log (1+T / \epsilon)}\right)^{1 /(n-1)}\right)=: \phi_{1},
$$

and thus the claim holds.

By the claim, there exists a component $U_{1}$ of $f^{-1}\left(Q\left(z_{1}, \phi_{1}\right)\right) \subseteq B(x, \epsilon)$, which is a normal domain, so that $\mu\left(f, U_{1}\right) \leq m / 2$. By repeating the previous arguments at most $M$ times, where $M$ is the largest integer so that $M \leq \log _{2} m$, we get a chordal 
ball $Q=Q\left(z_{M}, \phi_{M}\right)$ so that $f^{-1}(Q)$ has a component $U_{M}$ with $\mu\left(f, U_{M}\right)=1$. Hence $f$ restricted to $U_{M}$ is a homeomorphism onto $Q$.

\section{ACKNOWLEDGMENTS}

We thank Professors Nurlan Dairbekov, Juha Heinonen, Ilkka Holopainen, Pekka Koskela and Pekka Pankka for discussions, and Koskela for reading the manuscript. We also thank the referee for carefully reading the paper and for valuable comments.

\section{REFERENCES}

[1] L. Ahlfors: Conformal Invariants, McGraw-Hill, New York, 1973. MR 50:10211

[2] A. Bloch: Les théorèmes de M. Valiron sur les fonctions entières et la théorie de l'uniformisation, Ann. Fac. Sci. Toulouse, 17 (1925).

[3] M. Bonk and A. Eremenko: Covering properties of meromorphic functions, negative curvature and spherical geometry, Ann. of Math. (2), 152 (2000), no. 2, 551-592. MR 2002a:30050

[4] A. V. Chernavskii: Finite-to-one open mappings of manifolds, Mat. Sb., 65 (1964), 357369, 66 (1964), 471-472; English transl., Amer. Math. Soc. Transl. (2) 100 (1972), 253-267, 268-270. MR 30:2476. MR 36:3320

[5] A. Dress: Newman's theorem on transformation groups, Topology, 8 (1969), 203-207. MR 38:6629

[6] A. Eremenko: Bloch radius, normal families and quasiregular mappings, Proc. Amer. Math. Soc., 128 (2000), no. 2, 557-560. MR 2000e:30069

[7] J. Heinonen: The branch set of a quasiregular mapping, Proceedings of the ICM Beijing, Higher Education Press, Beijing, 2002, 691-700. MR 2003k:30034

[8] P. Koskela, J. Onninen, and K. Rajala: Mappings of finite distortion: Injectivity radius of a local homeomorphism, Preprint 266, Department of Mathematics and Statistics, University of Jyväskylä, 2002.

[9] O. Lehto and K. I. Virtanen: Quasiconformal mappings in the plane, Springer-Verlag, New York, 1973. MR 49:9202

[10] O. Martio, U. Srebro, and J. Väisälä: Normal families, multiplicity and the branch set of quasiregular mappings, Ann. Acad. Sci. Fenn. Math., 24 (1999), no. 1, 231-252. MR 99m:30043

[11] L. F. McAuley and E. E. Robinson: On Newman's theorem for finite-to-one open mappings on manifolds, Proc. Amer. Math. Soc., 87 (1983), no. 3, 561-566. MR 84d:57007

[12] D. Minda: Bloch constants for meromorphic functions, Math. Z., 181 (1982), 83-92. MR 84b:30033

[13] R. Miniowitz: Normal families of quasimeromorphic mappings, Proc. Amer. Math. Soc., 84 (1982), no. 1, 35-43. MR 83c:30026

[14] M. H. A. Newman: A theorem on periodic transformations of spaces, Quart. J. Math. Oxford Ser., 2 (1931), 1-9.

[15] Yu. G. Reshetnyak: Space Mappings with Bounded Distortion, Translations of Mathematical Monographs, vol. 73, Amer. Math. Soc., Providence, RI, 1989. MR 90d:30067

[16] S. Rickman: Quasiregular mappings, Springer-Verlag, New York, 1993. MR 95g:30026

[17] G. Valiron: Recherches sur le théorème de M. Picard, Ann. Sci. Ecole Norm. Sup., 38 (1921), 389-430.

[18] J. Väisälä: Discrete open mappings on manifolds, Ann. Acad. Sci. Fenn. Ser. A I, 392 (1966), 1-10. MR 34:814

[19] J. Väisälä: Lectures on n-dimensional quasiconformal mappings, Lecture Notes in Mathematics, Vol. 229, Springer-Verlag, Berlin and New York, 1971. MR 56:12260

[20] L. Zalcman: A heuristic principle in complex function theory, Amer. Math. Monthly, 82 (1975), 813-817. MR 52:757

Department of Mathematics and Statistics, University of Jyvëskylä, P.O. Box 35 (MAD), FIN-40014, University of Jyväskylä, Finland

E-mail address: kirajala@maths.jyu.fi 